\title{
Low-Power Optically Controlled Patch Antenna of Reconfigurable Beams
}

\author{
Deshuang Zhao, Yifei Han, Feng Liang, Qiaoli Zhang, and Bing-Zhong Wang \\ School of Physical Electronics, University of Electronic Science and Technology of China, Chengdu 610054, China \\ Correspondence should be addressed to Deshuang Zhao; dszhao@uestc.edu.cn
}

Received 22 June 2014; Accepted 14 August 2014; Published 28 August 2014

Academic Editor: J. S. Mandeep

Copyright ( 2014 Deshuang Zhao et al. This is an open access article distributed under the Creative Commons Attribution License, which permits unrestricted use, distribution, and reproduction in any medium, provided the original work is properly cited.

\begin{abstract}
A novel compact beam-reconfigurable patch antenna based on light control of no more than $30 \mathrm{~mW}$ optical powers is successfully demonstrated. It consists of one T-shape driven patch and one slot-etched parasitic patch. A silicon dice is employed as the photoconductive switch that is bridged across the slot center for optical control of reconfigurable beams. The antenna greatly reduces the total optical powers required for reconfigurable beams. Such design is based on the fact that the current phase change of the parasitic patch is sensitive to the conductivity of the silicon dice. A few conductivity changes of the silicon dice induced by the optical light can lead to a big phase change of the parasitic patch currents, eventually resulting in reconfigurable beams with low optical power requirement.
\end{abstract}

\section{Introduction}

With rapid development of intelligent wireless systems, reconfigurable antenna has received increasing attention due to its compact size, reconfigurable frequencies [1,2], changeable beams [3, 4], and adaptive nulls [5-8]. Recently, photoconductive switch becomes very popular for reconfigurable antennas due to free DC-bias networks, perfect isolation of the controlling circuits from the microwave signals, remote control, high-power handling, and fast switch action [9-15].

Although the photoconductive switch has many attractive advantages, the drawback of high optical power requirement makes the optically reconfigurable antenna costly. Typically, more than $200 \mathrm{~mW} / \mathrm{mm}^{2}$ optical powers are required for a single microstrip photoconductive switch to achieve lower than $1 \mathrm{~dB}$ insert loss with isolations lower than $15 \mathrm{~dB}$ in $\mathrm{S}$ band [16]. The total optical powers increase as illumination dimensions plus the number of the photoconductive switches utilized in the antennas [11, 15]. In [11], a total of about $100 \mathrm{~mW}$ optical powers are required for two photoconductive switches. In [15], a reconfigurable antenna of four siliconbased photoconductive switches needs about $2 \mathrm{~W}$ optical powers. This will inevitably increase the cost of the optically controlled antennas because high-power fiber laser sources are usually quite expensive.

In this paper, an attempt is made to design a reconfigurable antenna that can be optically controlled with low optical powers. Our method is based on the fact that the current phase of a slotted parasitic patch loaded with a photoconductive switch is sensitive to the conductivity of the silicon dice. A little change in the conductivity of the silicon dice induced by the light can lead to a big change of the current phase of the slotted parasitic patch. Thus, we can employ a few optical powers to activate the photoconductive switch to change the current phase of the parasitic patch. Another advantage of this method is that the attenuation caused by the photoconductive switch is very small. This is because the photoconductive switch under low power illumination operates in the state of high resistance and few currents pass through the switch. In [8], we have reported the results but did not present the mechanism of this antenna. In this paper, we detail the principle of phase changes of the parasitic patch that is optically controlled by the fiber laser. With the proposed method, we successfully demonstrate a reconfigurable patch antenna with no more than $30 \mathrm{~mW}$ optical powers. This antenna greatly reduces the total optical 
powers required for beam reconfiguration. Both simulations and measurements demonstrate the performance of the proposed antenna.

\section{Antenna Design}

Figure 1 shows the geometrical structure of the proposed antenna. It is designed to operate within the S-band. To reduce the antenna size, two patch elements are used. They are closely spaced to each other with an interval of $s=6.5 \mathrm{~mm}$, as shown in Figure 1(a). The larger one with T-shape geometry acts as the driven element. This patch is electrically fed by a coaxial probe, located at the patch center in the $y$-axis direction and a distance $q=6 \mathrm{~mm}$ away from the outer edge of the patch in the $x$-axis direction. The two lateral wings of the T-shape driven patch are designed to match the antenna for the same working band at different switch states (on and off). The driven patch has dimensions of $a=48 \mathrm{~mm}, b=$ $69 \mathrm{~mm}, c=22 \mathrm{~mm}, d=30 \mathrm{~mm}$, and $e=10.5 \mathrm{~mm}$. The smaller square patch neighboring the T-shape driven patch serves as the passive parasitic element. It has a size of $f=41.6 \mathrm{~mm}$ and $g=41.6 \mathrm{~mm}$ with a slot $(l=28.5 \mathrm{~mm}$ and $m=1.5 \mathrm{~mm})$ etched in the patch center. A single switch is installed in the slot center by using silver epoxy. The antenna is fabricated on a single-sided FR4 substrate of dimensions $125 \mathrm{~mm} \times 125 \mathrm{~mm}$ $\times 1 \mathrm{~mm}$ and mounted above a metal ground with a spacing of $4.46 \mathrm{~mm}$, as shown in Figure 1(b). The total size of the antenna is about $0.9 \lambda \times 0.9 \lambda \times 0.06 \lambda$, where $\lambda$ is the center wavelength at $2.2 \mathrm{GHz}$.

\section{Photoconductive Switch}

The switch used in the proposed antenna is made of a $2 \mathrm{~mm}$ $\times 1 \mathrm{~mm} \times 0.28 \mathrm{~mm}$ high-resistivity silicon dice with a dark resistivity of $3000 \Omega \cdot \mathrm{cm}$ and a relative permittivity of $\varepsilon_{r}=$ 11.9. In simulations, the photoconductive switch is modeled as an insulator in the state of no light. When the laser is turned on, the photoconductive switch is modeled as a conductive silicon dice of finite conductivity. The conductivity of the silicon dice is calculated with the formulas proposed in [17, 18].

The switch behavior of the silicon dice is based on the photoconductive effects that the silicon conductivity increases under the illumination of light whose photon energy is higher than the silicon band gap value $(1.1 \mathrm{eV})$. When the light is turned off, the silicon dice acts as an insulator. When the light is turned on, the silicon dice behaves like a lossy metal line whose conductivity increases as the incident optical power density. Increasing optical powers is an effective method to reduce the insertion loss. However, high optical powers not only require expensive laser sources but also result in many harmful nonlinear photoconductive effects [18].

In order to reduce optical power, we explore the use of the high-resistivity states of the photoconductive switch to form reconfigurable beams. This method is based on the fact that the resonance of the switch-loaded parasitic patch is sensitive to the conductivity of the silicon dice. By numerical simulations of the isolated parasitic patch loaded with the photoconductive switch, we find that a little change of the conductivity $\sigma$ of the silicon dice in the range of $0 \mathrm{~S} / \mathrm{m}$ to $100 \mathrm{~S} / \mathrm{m}$ can lead to a big change of the resonance of the parasitic patch, as shown in Figure 2. Thus, we can employ low optical powers to activate the switch for reconfigurable beams.

Besides that, another benefit of this method is that the attenuation introduced by the switch can be neglected. This is because the switch acts like an insulator when it operates in high-resistivity states. Almost no currents pass through the switch. Most currents go through the sided metal edges of the slot.

\section{Reconfigurable Beam Forming}

The proposed antenna can be regarded as a two-element array. With the concept of the active element pattern (AEP) proposed in [19], the E-plane radiation pattern of the antenna can be expressed by a sum of the AEPs of the driven patch and the parasitic patch as follows:

$$
F(\theta)=F_{d}(\theta)+\frac{\left|I_{p}\right|}{\left|I_{d}\right|} F_{p}(\theta) e^{j\left(k \Delta d \sin \theta+\Delta \varphi_{a}(\theta)+\Delta \varphi_{I}\right)},
$$

where $F_{d}(\theta)$ and $F_{p}(\theta)$ denote the AEPs of the driven patch and the parasitic element, respectively. $\Delta \varphi_{a}(\theta)$ is the angledependent phase difference of $F_{p}(\theta)$ relative to $F_{d}(\theta) . \Delta \varphi_{I}$ is the phase difference of the current $I_{p}$ induced on the parasitic element relative to the current $I_{d}$ excited on the driven patch. The parameter $k$ is the wave number, $\Delta d$ is the center spacing of the two patches, and $\theta$ is the view angle from the $z$-axis.

Based on the array theory, the reconfigurable beams can be realized by changing the intensity of $I_{p}, I_{d}$ or their phase difference $\Delta \varphi_{I}$, or both of them. If we let $\left|I_{p}\right| /\left|I_{d}\right|$ satisfy

$$
F_{d}\left(\theta_{0}\right)=\frac{\left|I_{p}\right|}{\left|I_{d}\right|} F_{p}\left(\theta_{0}\right)
$$

and alter the phase difference $\Delta \varphi_{I}$ of the currents on the driven and parasitic patches, beam patterns with tunable null depth at the angle $\theta_{0}$ can be achieved.

In this paper, $\Delta \varphi_{I}$ is changed by the extra phase $\Delta \varphi_{e}$ of the currents introduced by the resonance shift of the switchloaded parasitic element. If we model the switch-loaded parasitic element with a LRC circuit, the extra phase $\Delta \varphi_{e}$ added to $\Delta \varphi_{I}$ can be estimated by

$$
\Delta \varphi_{e}=\tan ^{-1}\left[\frac{\left(\omega+\omega_{p}\right) L_{p}\left(\omega-\omega_{p}\right)}{\left(\omega^{2} R_{p}\right)}\right] .
$$

The above formula can be derived from the impedance formula of the RLC circuit

$$
\begin{aligned}
Z & =R_{p}+j\left(\omega L_{p}-\frac{1}{\omega C_{p}}\right) \\
& =R_{p}+j \frac{\left(\omega+\omega_{p}\right) L_{p}}{\omega^{2}}\left(\omega-\omega_{p}\right),
\end{aligned}
$$




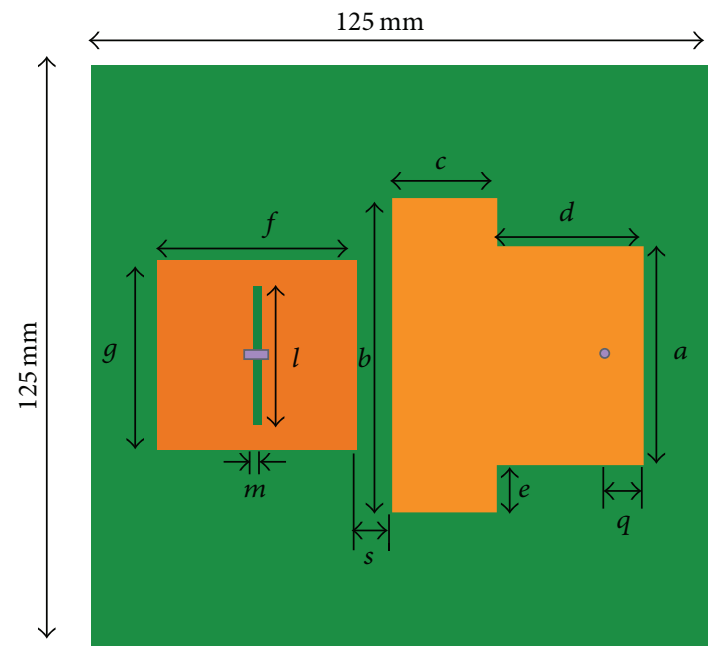

(a) Top view

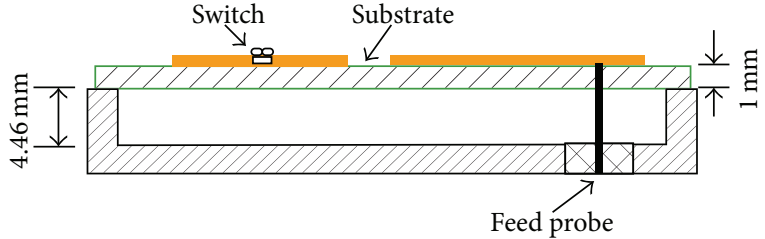

(b) Sectional view

FIGURE 1: The geometrical structure of the proposed antenna.

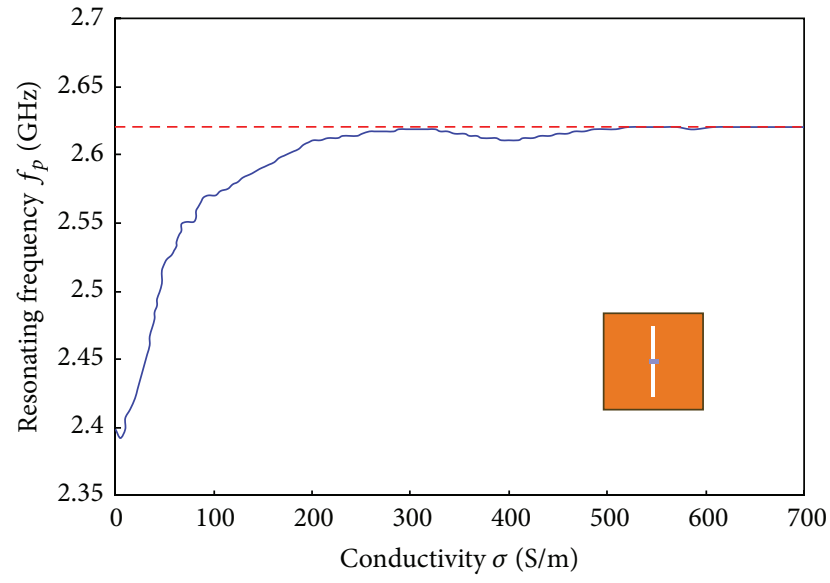

FIGURE 2: The changes of the resonating frequency of the isolated parasitic element with respect to the conductivity $\sigma$ of the silicon dice.

where $R_{p}, L_{p}$, and $C_{p}$ are the resistance, inductance, and capacitance of the equivalent circuit of the switch-loaded parasitic element, respectively. The symbol $\omega$ is the angular frequency of the signal, and $\omega_{p}=1 / \sqrt{L_{p} C_{p}}$ is the resonance of the parasitic element. It can be found from (3) that a little shifting of $\omega_{p}$ from $\omega$ can lead to an extra phase $\Delta \varphi_{e}$. Thus, tunable null depth can be achieved by dynamically adjusting the current phase of the parasitic element with the optical power.

\section{Impedance Match in Different Switch States}

In order to achieve good impedance match in different switch states, two lateral wings are added to the originally squared driven patch. This structure provides an effective way to control the impedance bandwidth of the proposed antenna

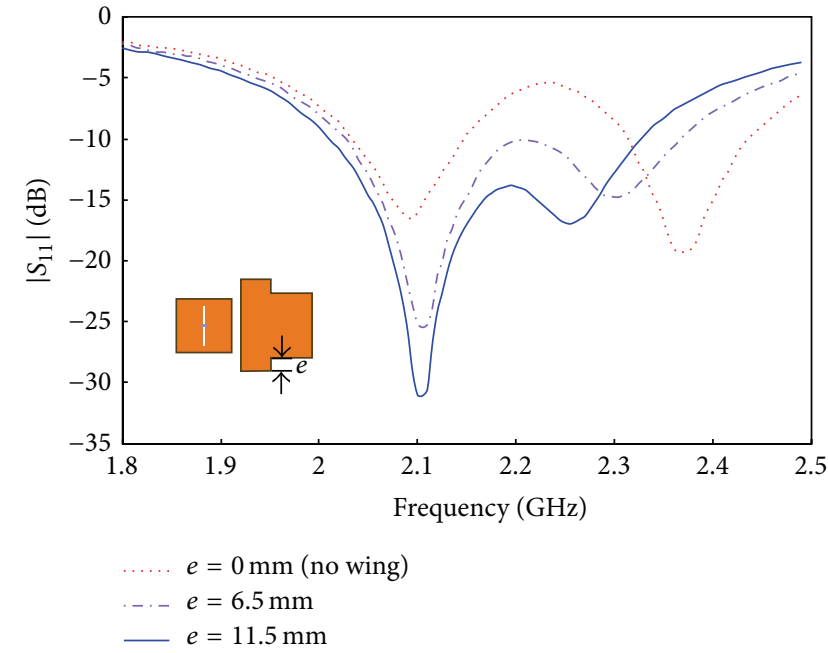

FIGURE 3: The impacts of the wing length on the resonances of the T-shape driven patch and the switch-loaded element without optical illumination.

by changing the wing length. Figure 3 depicts the impacts of the wing length on the resonances of the parasitic element and the driven patch. We see that the spacing between the resonating frequency $\left(f_{p}\right)$ of the parasitic element and that $\left(f_{d}\right)$ of the driven patch can be adjusted by tuning the wing length. By properly designing $f_{p}$ and $f_{d}$, the antenna exhibits a broad bandwidth. We also find that the wing length can lower $f_{p}$ while keeping $f_{d}$ almost unchanged. This feature is very important for impedance match since it allows us to place $f_{p}$ close to $f_{d}$ by optimizing the wing length in the state of no light. As long as the increased resonance of the parasitic element caused by the optical power incident on the photoconductive switch does not exceed the maximum impedance bandwidth offered by the wing structure, the 
antenna can achieve good impedance match in different switch states.

\section{Simulated and Measured Results}

Both simulations and measurements are performed to validate the proposed design. The simulations are carried out with Ansoft HFSS. The return losses of the antenna are measured with Agilent E8361A, and the radiation measurement is completed in an anechoic chamber. The antenna is controlled by a laboratory fiber-coupled continuous-wave semiconductor laser, which is packaged into a metal box, as shown in Figure 4. The feeding fiber is installed above the photoconductive switch with a transparent Plexiglass holder of $\varepsilon_{r}=3.0$.

Figure 5 shows the measured and simulated return losses of the proposed antenna. A good agreement is achieved between simulations and measurements. It can be found that the proposed antenna has a $10 \mathrm{~dB}$ bandwidth of $190 \mathrm{MHz}$ ranging from $2.10 \mathrm{GHz}$ to $2.29 \mathrm{GHz}$ in the state of no light illumination and a $10 \mathrm{~dB}$ bandwidth of $200 \mathrm{MHz}$ ranging from $2.05 \mathrm{GHz}$ to $2.25 \mathrm{GHz}$ in the state of $50 \mathrm{~mW}$ optical powers incident on the switch. The proposed antenna exhibits approximately equal impedance bandwidth in different switch states, showing a good impedance match. By tuning the optical power from zero to $50 \mathrm{~mW}$, the measured impedance bandwidth keeps almost unchanged. This feature is very essential in practical applications since most wireless systems require reconfigurable beams without changing the impedance bandwidth.

Figure 6(a) depicts the simulated radiation patterns of the proposed antenna in the E-plane obtained by using different silicon conductivity. Figure 6(b) shows the measured beams in the E-plane under different optical power illumination. As expected in the previous sections, tunable null depth is successfully achieved by controlling the optical powers incident on the photoconductive switch. In the state of no light, both simulations and measurements show that a null is generated but not the deepest one. This null generates from the initial phase difference between the parasitic element and the driven patch, caused by the mutual coupling effects. Since we need a fixed null away from the broadside direction, the fields radiated by the two patches should have equal intensity be out-of-phase. In the off state, we find that the currents of the two patches are not out-of-phase since the initial null is not the deepest one. To get the null deeper, we can activate the photoconductive switch with the optical powers to change the resonance of the parasitic element and introduce an extra current phase to the parasitic element. It can be seen from Figure 6 that the deepest null is obtained by increasing the silicon conductivity up to $4 \mathrm{~S} / \mathrm{m}$ or by raising the optical powers up to about $1 \mathrm{~mW}$. To further increase the conductivity or raise the incident optical powers, the null becomes shallow. Gradually, the null disappears at $50 \mathrm{~S} / \mathrm{m}$ or nearby $30 \mathrm{~mW}$ optical powers. This is because the additional phase makes the current phase difference between the two patches exceed $\pi$. With the proposed antenna, we find that, by switching the optical powers between $1 \mathrm{~mW}$ and $30 \mathrm{~mW}$, the antenna can

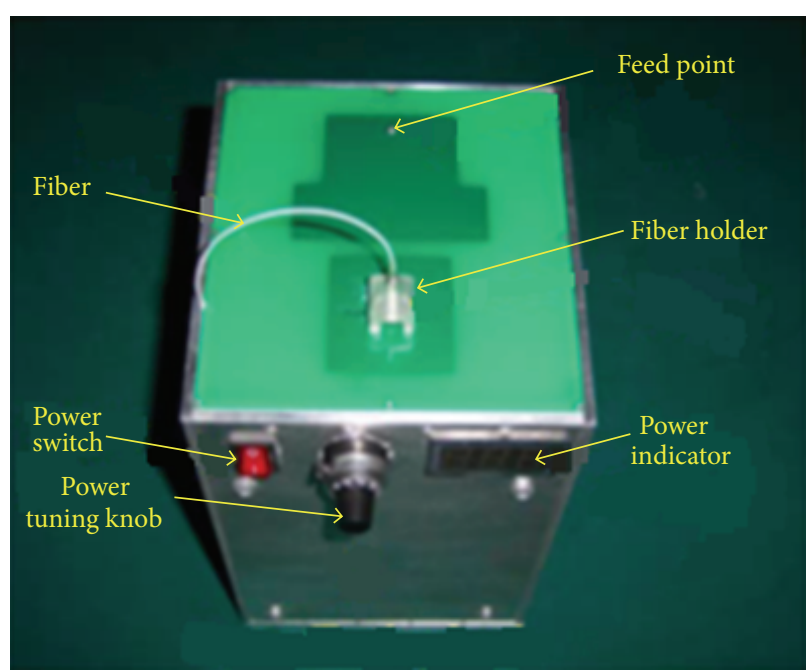

Figure 4: The photo of the proposed antenna.

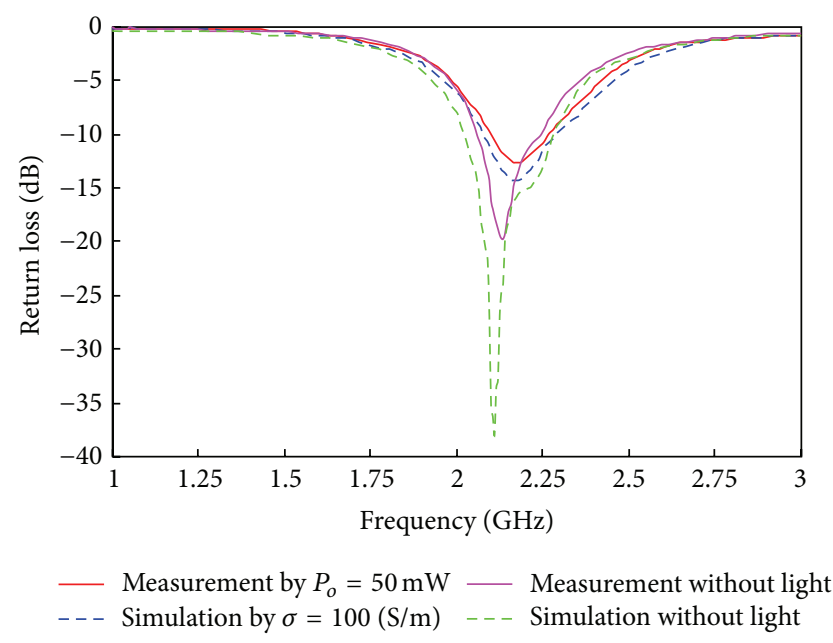

FIGURE 5: The comparison of the return losses of the proposed antenna operating in different switch states.

adaptively reconfigure its radiation beams with a deep null beam or a beam of no nulls for interference detection and suppression. This is an exciting result since the maximum optical powers required for the null depth tuning are no more than $30 \mathrm{~mW}$.

The measured gains of the proposed antenna are $6.8 \mathrm{~dB} @ 2.2 \mathrm{GHz}$ by using $50 \mathrm{~mW}$ optical powers to activate the photoconductive switch, $5.5 \mathrm{~dB} @ 2.2 \mathrm{GHz}$ by using $1 \mathrm{~mW}$ optical powers, and $5.5 \mathrm{~dB} @ 2.2 \mathrm{GHz}$ without light illumination. The slight reduction of the gain in the last two states is because the null causes some increase of the side lobe level, which can be seen from Figures 6(a) and 6(b). By removing the switch, the gain of the antenna is around $5.5 \mathrm{~dB} @ 2.2 \mathrm{GHz}$, showing that the losses caused by the photoconductive switch can be neglected. 


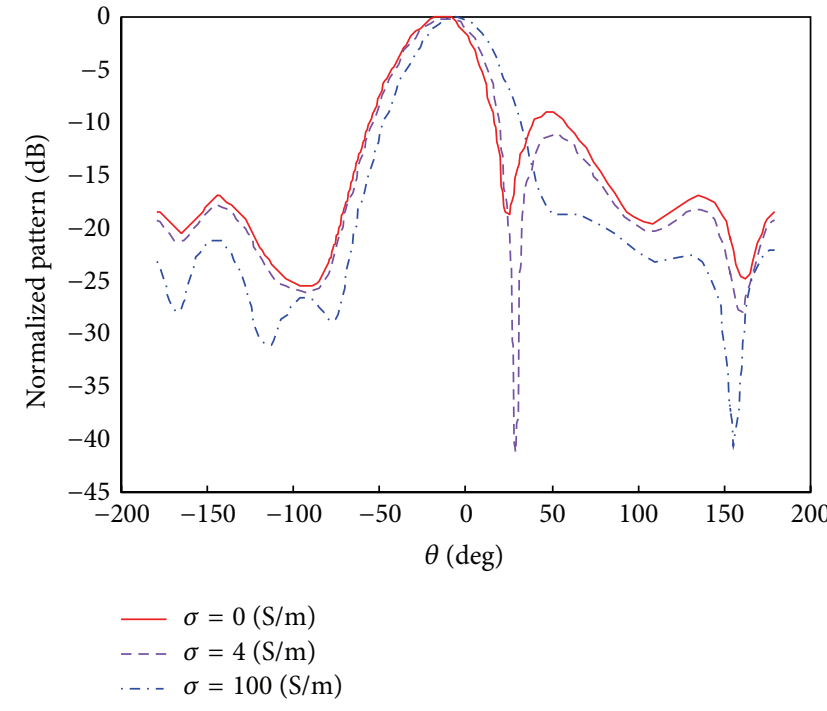

(a) The simulated beam pattern with different conductivity

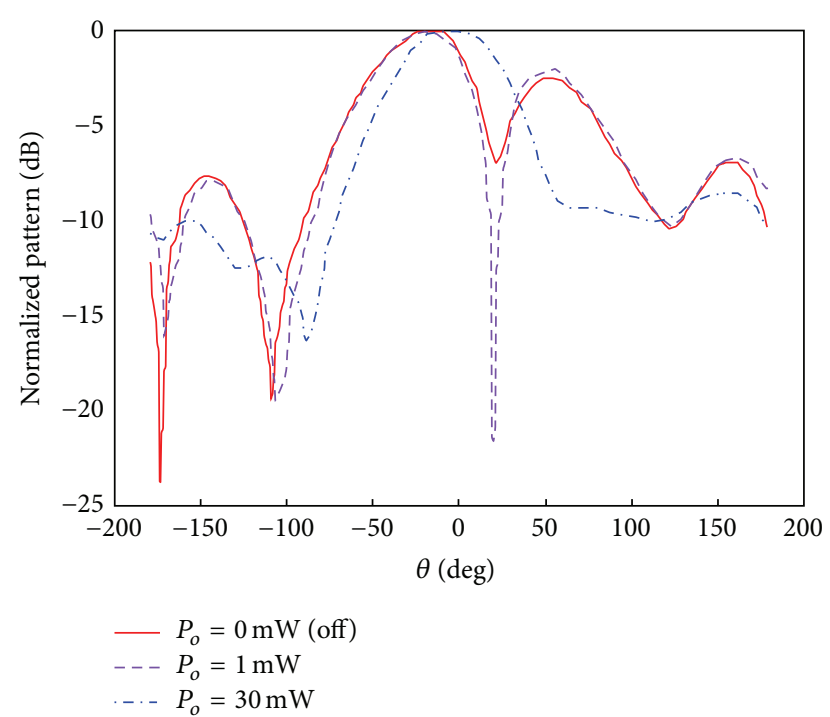

(b) The measured beam patterns with different optical powers

FIgURE 6: The reconfigurable beams in the E-plane. (a) The numerical simulations and (b) the measurements.

\section{Conclusion}

In this paper, we illustrate a design by using optically controlled method for reconfigurable beams with low optical power excitation. This design is implemented with a driven patch and a switch-loaded parasitic element. The single highresistivity photoconductive switch is loaded in the center of the slot that is etched in the parasitic element. The beamreconfigurable functionality relies on the fact that the current phase change of the parasitic patch is sensitive to the conductivity of the silicon dice. A few conductivity changes of the silicon dice induced by the optical light can lead to a big phase change of the parasitic patch currents, eventually resulting in reconfigurable beams with low optical power requirement. Both the simulated and measured results demonstrate that the developed antenna has good performance in reconfigurable-beam forming, feed impedance matching, and low optical power requirement. These advantages make the proposed antenna quite attractive for intelligent wireless applications such as interference detection and suppression, as well as target locating.

\section{Conflict of Interests}

The authors declare that there is no conflict of interests regarding the publication of this paper.

\section{Acknowledgments}

This work was supported by the National Natural Science Foundation of China (nos. 61371106 and 61331007) and the Fundamental Research Funds for the Central Universities (Grant no. ZYGX2011X008).

\section{References}

[1] H. F. Abutarboush, R. Nilavalan, S. W. Cheung et al., "A reconfigurable wideband and multiband antenna using dualpatch elements for compact wireless devices," IEEE Transactions on Antennas and Propagation, vol. 60, no. 1, pp. 36-43, 2012.

[2] A. H. Ramadan, K. Y. Kabalan, A. El-Hajj, S. Khoury, and M. Al-Husseini, "A reconfigurable U-Koch microstrip antenna for wireless applications," Progress in Electromagnetics Research, vol. 93, pp. 355-367, 2009.

[3] Y.-Y. Bai, S. Xiao, M.-C. Tang, Z.-F. Ding, and B.-Z. Wang, "Wide-angle scanning phased array with pattern reconfigurable elements," IEEE Transactions on Antennas and Propagation, vol. 59, no. 11, pp. 4071-4076, 2011.

[4] W. Kang, K. H. Ko, and K. Kim, "A compact beam reconfigurable antenna for symmetric beam switching," Progress in Electromagnetics Research, vol. 129, pp. 1-16, 2012.

[5] R. L. Haupt, J. Flemish, and D. Aten, "Adaptive nulling using photoconductive attenuators," IEEE Transactions on Antennas and Propagation, vol. 59, no. 3, pp. 869-876, 2011.

[6] S. Yong and J. T. Bernhard, "Reconfigurable null scanning antenna with three dimensional null steer," IEEE Transactions on Antennas and Propagation, vol. 61, no. 3, pp. 1063-1070, 2013.

[7] S. Yong and J. T. Bernhard, "A pattern reconfigurable null scanning antenna," IEEE Transactions on Antennas and Propagation, vol. 60, no. 10, pp. 4538-4544, 2012.

[8] D. Zhao, Y. Han, L. Lan et al., "Optically controlled microstrip patch antenna with reconfigurable null beams," Tech. Rep. APS/URSI, IEEE, Memphis, Tenn, USA, 2014.

[9] E. K. Kowalczuk, C. J. Panagamuwa, R. D. Seager, and J. C. Vardaxoglou, "Characterising the linearity of an optically controlled photoconductive microwave switch," in Proceeding of the 6th Loughborough Antennas and Propagation Conference (LAPC '10), pp. 597-600, Loughborough, UK, November 2010.

[10] C. J. Panagamuwa, A. Chauraya, and J. C. Vardaxoglou, "Frequency and beam reconfigurable antenna using photoconducting switches," IEEE Transactions on Antennas and Propagation, vol. 54, no. 2, pp. 449-454, 2006. 
[11] Y. Tawk, J. Costantine, S. Hemmady, G. Balakrishnan, K. Avery, and C. G. Christodoulou, "Demonstration of a cognitive radio front end using an optically pumped reconfigurable antenna system (OPRAS)," IEEE Transactions on Antennas and Propagation, vol. 60, no. 2, pp. 1075-1083, 2012.

[12] Y. Tawk, A. R. Albrecht, S. Hemmady, G. Balakrishnan, and C. G. Christodoulou, "Optically pumped frequency reconfigurable antenna design," IEEE Antennas and Wireless Propagation Letters, vol. 9, pp. 280-283, 2010.

[13] Y. Tawk, M. Bkassiny, G. El-Howayek, S. K. Jayaweera, K. Avery, and C. G. Christodoulou, "Reconfigurable front-end antennas for cognitive radio applications," IET Microwaves, Antennas and Propagation, vol. 5, no. 8, pp. 985-992, 2011.

[14] R. N. Lavallee and B. A. Lail, "Optically-controlled reconfigurable microstrip patch antenna," in Proceedings of the IEEE International Symposium on Antennas and Propagation, pp. 14, July 2008.

[15] G. P. Jin, D. L. Zhang, and R. L. Li, "Optically controlled reconfigurable antenna for cognitive radio applications," Electronics Letters, vol. 47, no. 17, pp. 948-949, 2011.

[16] L. Lan, D. Zhao, F. Liang, Q. Zhang, and B.-Z. Wang, "Influence of laser wavelength on insertion loss of silicon-based optically controlled microwave switch," Microwave and Optical Technology Letters, vol. 55, no. 1, pp. 187-190, 2013.

[17] W. Platte, "Effective photoconductivity and plasma depth in optically quasi-CW controlled microwave switching devices," IEE Proceedings, vol. 135, no. 3, pp. 251-254, 1988.

[18] D. D. Smith, J. F. Knudsen, and S. C. Moss, "Nonlinear optoelectronic effects in ultrafast photoconductive switches," in Interconnection of High Speed and High Frequency Devices and Systems, Proceedings of SPIE, pp. 146-161, September 1988.

[19] D. M. Pozar, “The active element pattern," IEEE Transactions on Antennas and Propagation, vol. 42, no. 8, pp. 1176-1178, 1994. 

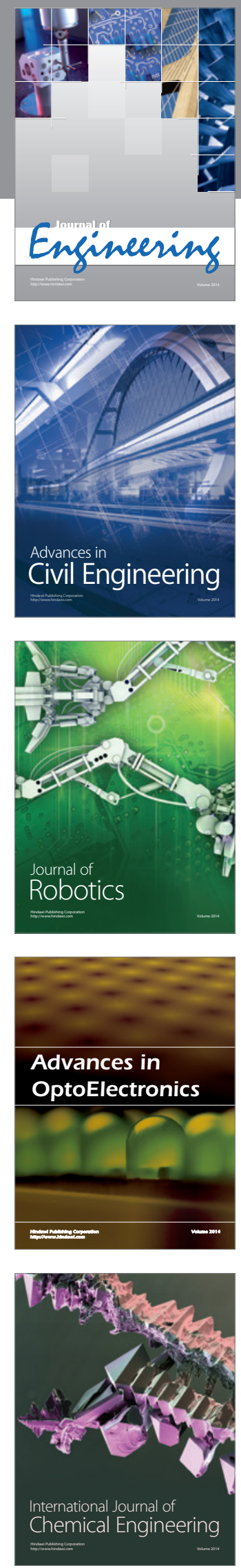

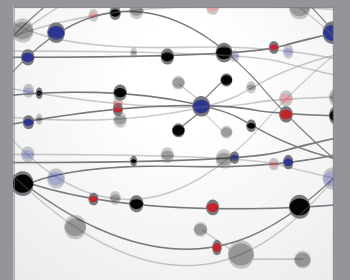

The Scientific World Journal
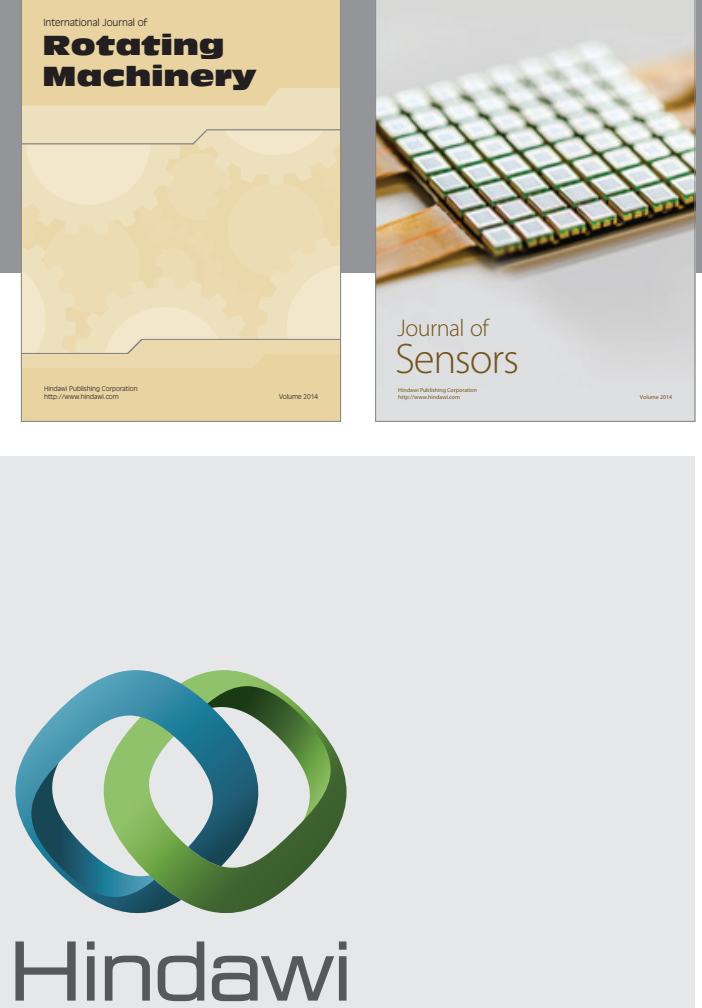

Submit your manuscripts at http://www.hindawi.com
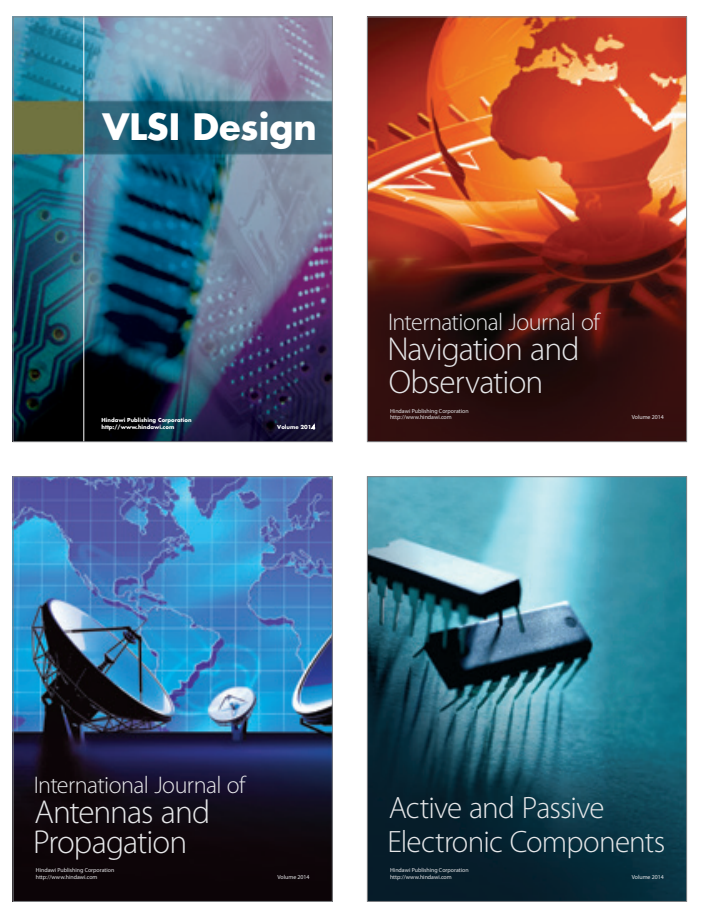
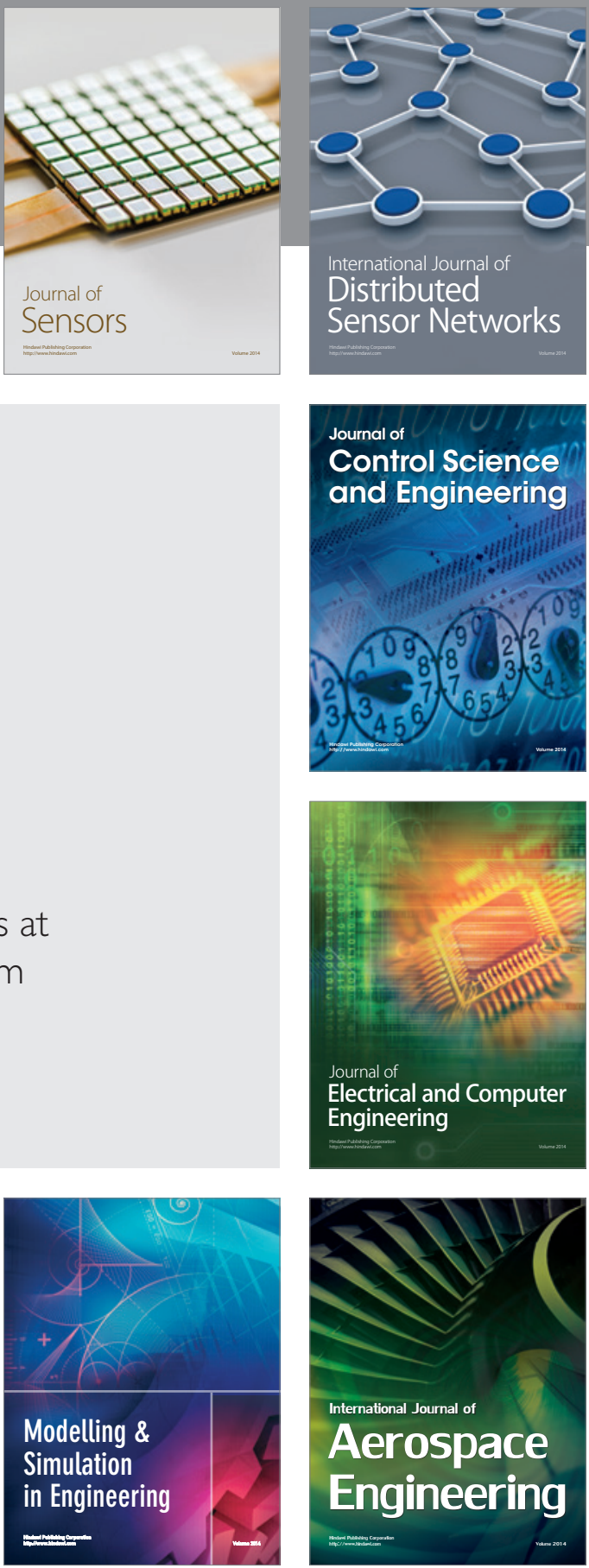

Journal of

Control Science

and Engineering
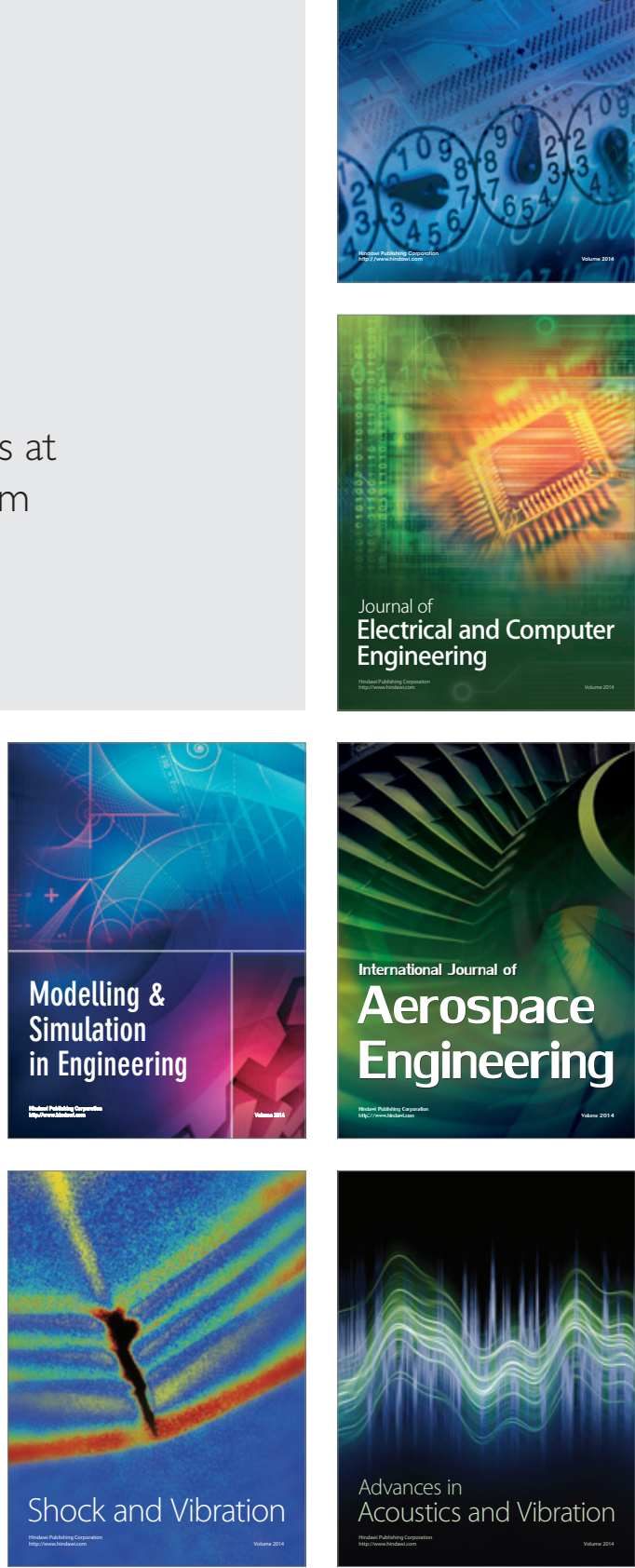